\title{
Pull-Type Radiologically Inserted Gastrostomy: An Improvised Technique Using a Frugal Innovation
}

\author{
Anurag Chahal ${ }^{1}$ Sundeep Malla ${ }^{1}$ Chinmaya Dash ${ }^{2} \quad$ Deepak Gupta $^{2} \quad$ Shivanand Gamanagatti ${ }^{1}$ \\ ${ }^{1}$ Department of Radiology, Jai Prakash Narayan Apex Trauma Centre, \\ AlIMS, New Delhi, India \\ 2Department of Neurosurgery, Jai Prakash Narayan Apex Trauma \\ Centre, AlIMS, New Delhi, India

\begin{abstract}
Address for correspondence Shivanand Gamanagatti, MD, centre, AlIMS, New Delhi 110029, India (e-mail: shivanandgamanagatti@rediffmail.com).
\end{abstract} \\ Department of Radiology, Jai Prakash Narayan Apex Trauma
}

J Clin Interv Radiol ISVIR 2019;3:7-11

Abstract


Keywords
- gastrostomy
- traumatic brain injury
- frugal innovation
- pull-through
gastrostomy

Purpose To describe a new technique for "pull type" radiologically inserted gastrostomy (RIG) and prospectively compare it with conventional (described) technique in terms of technical ease (fluoroscopy time, radiation dose) and safety profile.

Materials and Methods Adult patients with head injury with Glasgow coma scale (GCS) (M score) $<5$, one week after decompression or those requiring nutritional support for $>4$ weeks, or patients with recurrent aspiration pneumonitis on nasogastric feeding were included. Hemodynamically unstable patients or those with uncorrectable coagulopathy were excluded. Patients underwent pull-type RIG with alternate patient getting allocated to groups $\mathrm{A}$ (conventional technique) and B (new technique). The authors compared the technical success, complication rate, fluoroscopy time, radiation dose, and cost of hardware in these two groups.

Results The average fluoroscopy time for group A (9 patients) was $498.7 \pm 145.3$ seconds, whereas for group $B$ ( 16 patients), it was $302.8 \pm 54.1$ seconds with $p$-value $=0.06$. The mean radiation dose of group $A$ was $74.7 \pm 15.7 \mathrm{mGy}$, whereas for group $B$, it was $56.7 \pm 14.1 \mathrm{mGy}(p$-value $=0.004)$. The minor complication rates were $11.1 \%$ in both the groups.

Conclusion The authors' innovative technique using a customized snare has the potential to increase the technical ease of pull-type RIG with reduced fluoroscopy time, radiation dose, and cost with a similar safety profile.

\section{Introduction}

In patients with head injury, adequate and early (within 48 hours) nutritional supplementation improves the immediate clinical course and may improve neurologic outcome at 3 months. ${ }^{1,2}$ Early gastrostomy $(<24$ hours of intubation) is associated with a lower frequency of ventilator-associated pneumonia compared with a nasogastric (NG) tube in mechanically ventilated patients with head injury. ${ }^{3}$ Gastrostomy has been established as the method of choice for enteral feeding in patients with head injury. ${ }^{4}$ As opposed to percutaneous endoscopic gastrostomy that is well established, radiologically inserted gastrostomy (RIG) has evolved as a very useful but underused technique primarily due to limited availability of expertise. There

received

September 25, 2018

accepted

October 16, 2018

published online

March 27, 2019 are two predominant techniques of RIG: push and pull (peroral) techniques. Push technique usually uses smallor medium-bore tubes and requires gastropexy for large bore ( $>20 \mathrm{~F}$ ) gastrostomy tubes. Push tubes also have a higher peritoneal placement rate, due to inadvertent loss of apposition of the stomach to abdominal wall during serial dilatation of stoma. ${ }^{5}$ The per-oral pull technique with mushroom-shaped silicon disc retainers combines the benefits of endoscopic technique, by being wide bore (typically 24F) and radiologic techniques, owing to its higher success rate. ${ }^{6}$ Additionally, they are very secure and long lasting because of their fixed retention mechanism, which cannot be unlocked or deflated. ${ }^{7}$ Pull technique has a lower complication rate but a higher fluoroscopy time as compared with push technique. ${ }^{8}$ Multiple improvisations 
over the originally described per-oral technique ${ }^{6}$ have been described to reduce the radiation dose and improve technical ease and success of the procedure. ${ }^{9,10}$ These improvisations would add to the overall cost of the procedure, requiring additional hardware. ${ }^{9}$ We devise a new technique using frugal hardware to make it technically easy by reducing radiation dose, fluoroscopy time, and hardware cost.

\section{Materials and Methods}

It was a retrospective study approved by the institute review board. Adult patients with head injury who underwent pull-type per-oral feeding gastrostomy from April 2017 till March 2018 were included. Two techniques were used for the pull-type feeding gastrostomy, and patients were grouped into groups A (conventional technique) and B (new technique) based on it. We retrospectively compared the technical success, complication rate, fluoroscopy time, radiation dose, and cost of hardware in these two groups.

\section{Protocol}

All patients were kept fasting for a minimum of 8 hours before procedure. A single dose of prophylactic intravenous cefazolin was given 30 minutes before the procedure. Agitated patients were sedated using midazolam $(0.02 \mathrm{mg} / \mathrm{kg})$ and fentanyl $(1 \mu \mathrm{g} / \mathrm{kg})$. All the patients had a cuffed tracheostomy tube in situ at the time of procedure with variable ventilation requirements. Patients usually had a $16 \mathrm{~F}$ NG tube placed bedside on admission. If there was a difficulty in securing an NG tube bedside, it was inserted under fluoroscopic guidance before the procedure. The inferior margin of left lobe of the liver was identified using ultrasound and marked on the skin surface. Transverse colon could usually be identified on fluoroscopy and could be displaced by adequate gastric distension. The likely stoma site in the left subcostal region was properly cleaned and draped. Conventional technique was adapted from Szymski et al. ${ }^{6}$

\section{New Technique}

A snare was prepared by passing the free ends of an extra long straight tip $260 \mathrm{~cm}$ long 0.035-in hydrophilic guidewire (GW; Terumo) from the distal open tip of a $16 \mathrm{~F}$ suction catheter and pulling out the ends of GW through the proximal end and securing them together with bandage, thus forming a wire loop that protrudes out from the tip of the suction catheter (Video 1). This wire loop has a large perimeter $>50 \mathrm{~cm}$, and the loop area can be adjusted by pulling the ends of GW back and forth acting as a snare

\section{Video 1}

A frugal customized snare was designed using $16 \mathrm{~F}$ suction catheter $(50 \mathrm{~cm})$ and 0.035 -in hydrophilic guidewire $(260 \mathrm{~cm})$. Online content is viewable at: https://www.thiemeconnect.com/products/ejournals/ html/10.1055/s-0039-1681124. if the loop was shortened all the way. This snare loop is 10 times larger than the snare provided in the PEG kit (perimeter $\sim 5 \mathrm{~cm}$ ), and thus it is easier to target under fluoroscopy. This whole apparatus with a shortened loop was inserted through the orogastric route into the distal esophagus. An oropharyngeal airway (Guedel's) was usually required for placing this tube due to masseter spasm in these patients. Stomach was insufflated with room air through the NG tube until the gastric curvature was $5 \mathrm{~cm}$ below the costal margin and until the margins of the stomach were smooth and convex with stomach wall touching the anterior abdominal wall on lateral fluoroscopic projections. The puncture site was chosen at the mid body of the stomach equidistant from both curvatures and the skin site is anesthetized using 1\% lignocaine. Snare is progressed across the GE junction just prior to the puncture to avoid gastric decompression by leakage of air via the residual lumen of suction catheter, and large snare loop is placed in the body of distended stomach (-Fig. 1, step 1). A $14 \mathrm{G}$ puncture cannula provided in the PEG 24 Pull-S (Cook Medical) kit was used with a thrust to ensure gastric wall puncture observed under lateral fluoroscopy targeting the large snare loop (-Fig. 2). After puncture, 2 to $3 \mathrm{~mL}$ air was aspirated to confirm intragastric position and the inner stylet was removed. The blue GW provided with the PEG kit was inserted and negotiated through the snare loop (-Fig. 1, step 2). The loop was then shortened by pulling the outer ends, and the blue wire was snared along into the $16 \mathrm{~F}$ suction catheter (-Fig. 1, step 3) followed by pulling out the entire assembly altogether. The 24G gastrostomy tube was then attached to the oral end of blue GW, and a knotless connection was made (-Fig. 1, step 4). The assembly was then pulled from the puncture end of blue GW under fluoroscopic guidance until its tip was opposed against the anterior wall of stomach (-Fig. 1, step 5). A deep stab 1.5 to $2 \mathrm{~cm}$ in size was given along the wire to take out the tapered end of the G-tube onto the skin surface and further pulled it till the inner mushroom bolster opposed the gastric wall to the abdominal wall. Another external fixation plate provided fixation on the cutaneous surface to keep the stoma site compressed (-Fig. 1, step 6). A gastropexy was not required because the technique obviates any push maneuvers. The tube position may be further confirmed by contrast injection under fluoroscopy. Patients were kept fasting for another 2 hours after the procedure, which was followed by $50 \mathrm{~mL}$ saline pushes 2 hourly for another 4 hours. Feeding with milk-based formula was started 6 hours after placement of gastrostomy tube. They were observed during their intrahospital course and 1 month after discharge for any tube-related complications. The complications were divided into major and minor complications according to the Society of Interventional Radiology (SIR) classification system. ${ }^{11}$

\section{Results}

Total 24 patients were included in the study, with 9 patients in group A and 15 in group B. The mean age of patients in group A was $36.2 \pm 4.9$ years (range: $23-61$ years), which was 

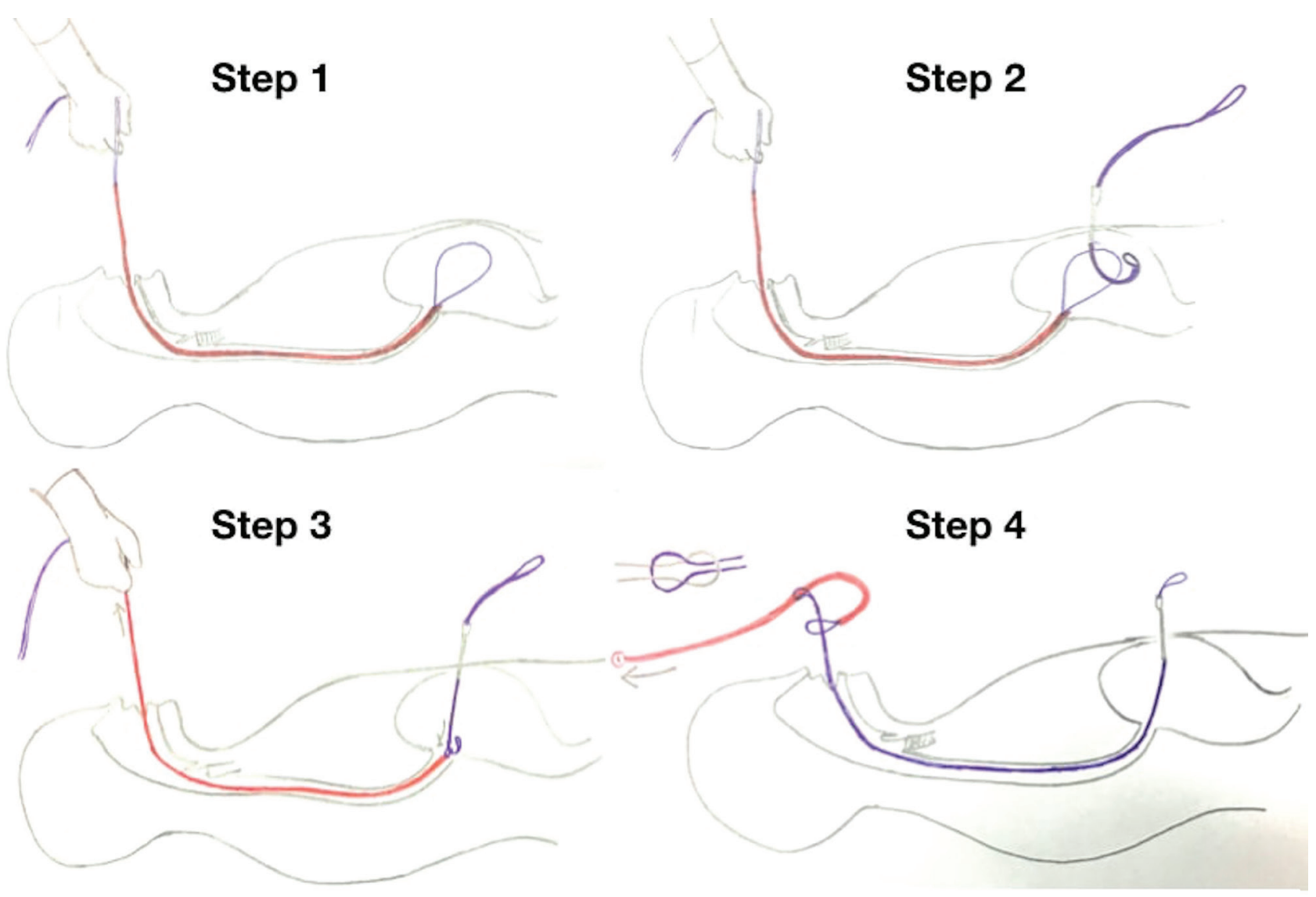

\section{Step 5}
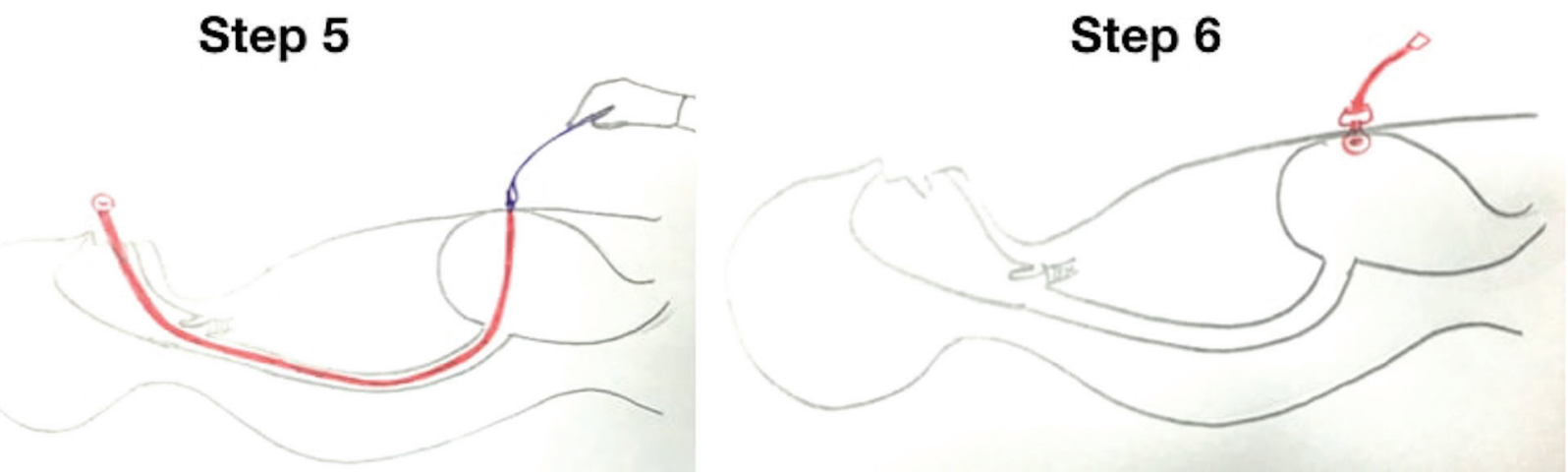

Fig. 1 The schematic diagram of the various steps of our new technique.

not significantly different from that in group B, which was $32.3 \pm 3.8$ years (range: $22-46$ years) $(p=0.26)$. All procedures were technically successful. Radiation dose in group B was less ( $56.7 \pm 14.1 \mathrm{mGy}$ ) as compared with group A $(74.7 \pm 15.7)$ with significant $p$ value ( $p=0.004)$. The fluoroscopy time in group B (302.8 \pm 54.1 seconds) was less than that in group A (498.7 \pm 145.3 seconds), and this difference was approaching significance $(p=0.06)$. One patient each in groups $A$ and $B$ had peristomal infection/pus discharge during inhospital follow-up. Each of these cases grew Pseudomonas on pus cultures and resolved with appropriate intravenous antibiotics. One patient in group A died on sixth day of the procedure due to unrelated causes. One patient in group B developed mild aspiration pneumonitis during postprocedure period, which could be attributable to their underlying illness and was not necessarily procedure related. It resolved over next 5 days on intravenous antibiotics. Thus, there were no major complications and only $9.1 \%$ minor complications in group A and 6.67\% in group B. Cost analysis revealed that cost of standard hardware for group $A$ patients (\$275) was much higher than that used in group B (\$150).

\section{Discussion}

Multiple techniques for obtaining GW access from the skin to the mouth have been reported for radiologically guided antegrade gastrostomy placement using a snare. One such technique uses a small-perimeter snare provided 


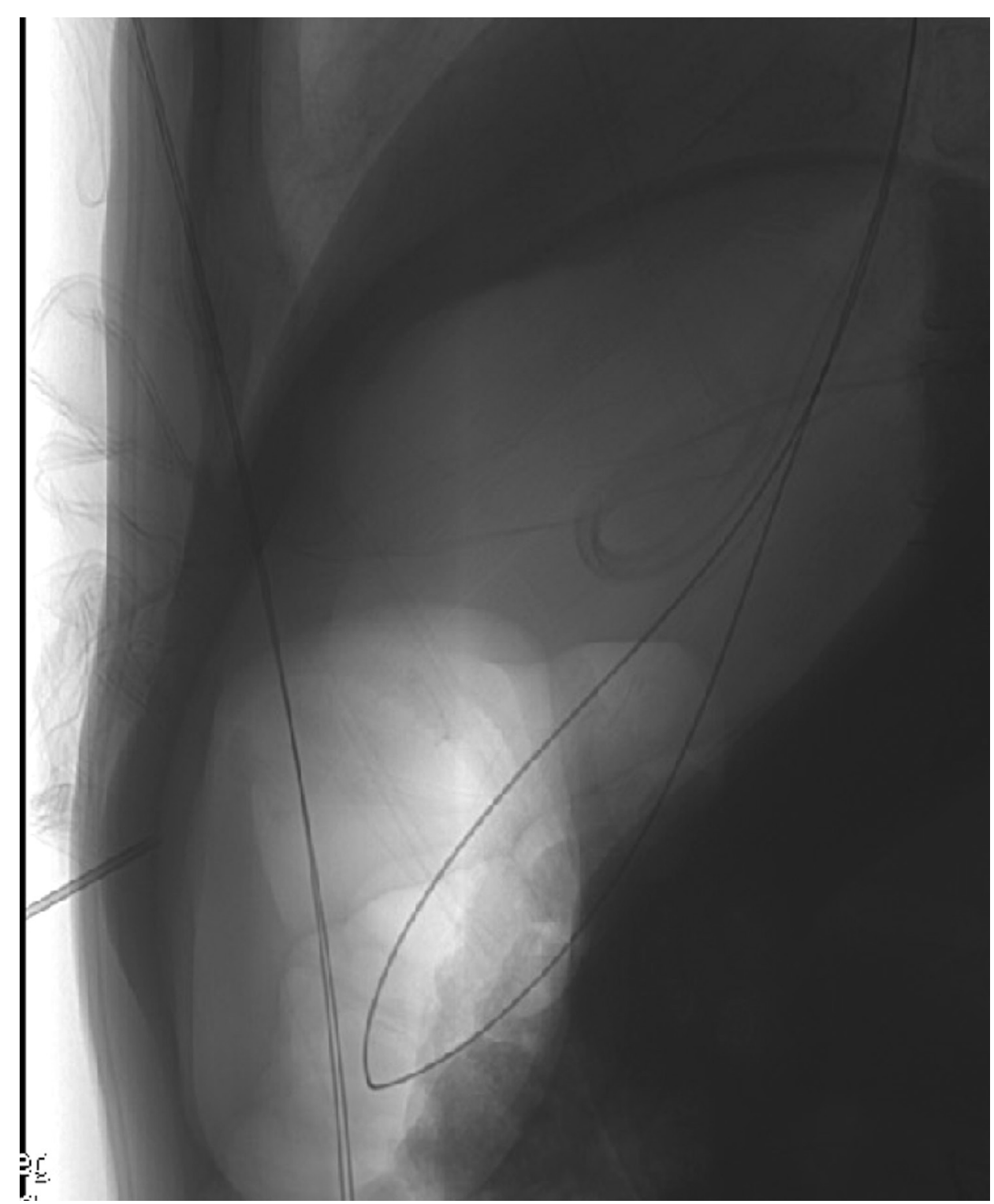

Fig. 2 Lateral fluoroscopic image of upper abdomen with a 14G puncture cannula at skin site targeting the loop of customized snare in the body of distended stomach with radiopaque tip of nasogastric tube seen in fundus of stomach.

in the PEG kit for snaring the GW..$^{10}$ We believe that the small perimeter of snare makes it technically difficult to target it, thus increasing the fluoroscopy time. Another improvisation described by Cantwell and Murray ${ }^{9}$ made a large diameter $(10 \mathrm{~cm})$ customized snare using a set of four 20-cm-long 0.018-in nitinol wires crimped both distally and proximally and annealing them by heating and then sterilizing it. The large size of the snare acts as an easy target for GW without the need for sheath insertion and GW and catheter manipulation. Our technique is a similar technique, but uses a very large snare loop (perimeter $\sim 50 \mathrm{~cm}$ ) that is uniformly effective and technically easier. As these patients often require multiple head computed tomographic (CT) scans for their primary disease, reducing the cumulative radiation dose is important. The higher radiation dose with conventional technique might be due to difficulty encountered in cannulation of the GE junction or in retrieving the catheter through the mouth of patients with head injury with significant masseter spasm. A previous study ${ }^{12}$ comparing push and pull gastrostomy quoted a fluoroscopy time of 6 minutes for pull gastrostomy, which is comparable to that with our novel technique but lesser than observed with conventional technique in our study. Our patient subset included patients with head injury, most of whom were ventilated with multiple problems such as rigidity and masseter spasm. They were difficult patients for a per-oral (pull) approach than conscious patients with head and neck cancers or neuromuscular disease included in previous studies. ${ }^{8}$ Cost reduction is a big factor in a resourcechallenged setting, and the new technique was effective in cutting the costs to almost half of the conventional technique. Thus this new technique retains all the advantages of the pullthrough technique with reduction in fluoroscopic time, cost, radiation dose, and thus technical difficulty. The minor complication rate was similar in the two methods 
ensuring safety of the new technique. To our knowledge, such improvisation has not been described as yet. Our study has a few limitations. First, this was a retrospective study and we cannot rule out any factors that might have influenced the interventional radiologist to choose one technique over the other, but we could not find any indications where one would be preferred over the other. Second, the sample size is limited and not robust to draw any conclusions. Third, we included patients with only a single indication for gastrostomy, that is, head injury, who usually had masseter spasm favoring our technique of snaring over the conventional retrograde technique. Patients having neurologic dysphagia due to other causes were not evaluated. Our technique needs to be applied to a wider and larger patient population. Additionally, we have had limited experience of performing per-oral pull-type gastrostomy since we were doing push-type gastrostomy before this and this might be one of the reasons for a higher fluoroscopy time. The essence of this study lies in using a frugal innovation to improvise a technical procedure reducing the procedure cost, procedure time, and radiation dose.

\section{Conclusion}

Our innovative technique of pull-type per-oral RIG using a large customized snare has the potential of improvising the procedure in terms of fluoroscopy time, radiation dose, and cost with a comparable safety profile.

\section{Conflict of Interest}

None.

\section{Ethical Approval}

All procedures performed in studies involving human participants were in accordance with the ethical standards of the institutional ethics committee and with the 1964 Helsinki declaration and its later amendments or comparable ethical standards.

\section{Informed Consent}

Informed consent was obtained from all individual participants included in the study.

\section{References}

1 Taylor SJ, Fettes SB, Jewkes C, Nelson RJ. Prospective, randomized, controlled trial to determine the effect of early enhanced enteral nutrition on clinical outcome in mechanically ventilated patients suffering head injury. Crit Care Med 1999;27(11):2525-2531

2 Perel P, Yanagawa T, Bunn F, Roberts I, Wentz R, Pierro A. Nutritional support for head-injured patients. Cochrane Database Syst Rev 2006;(4):CD001530

3 Kostadima E, Kaditis AG, Alexopoulos EI, Zakynthinos E, Sfyras D. Early gastrostomy reduces the rate of ventilatorassociated pneumonia in stroke or head injury patients. Eur Respir J 2005;26(1):106-111

4 D'Amelio LF, Hammond JS, Spain DA, Sutyak JP. Tracheostomy and percutaneous endoscopic gastrostomy in the management of the head-injured trauma patient. Am Surg 1994;60(3):180-185

5 Thornton FJ, Fotheringham T, Haslam PJ, McGrath FP, Keeling F, Lee MJ. Percutaneous radiologic gastrostomy with and without T-fastener gastropexy: a randomized comparison study. Cardiovasc Intervent Radiol 2002;25(6):467-471

6 Szymski GX, Albazzaz AN, Funaki B, et al. Radiologically guided placement of pull-type gastrostomy tubes. Radiology 1997;205(3):669-673

7 Funaki B, Zaleski GX, Lorenz J, et al. Radiologic gastrostomy placement: pigtail- versus mushroom-retained catheters. AJR Am J Roentgenol 2000;175(2):375-379

8 Han K, Kim MD, Kwon JH, et al. Randomized controlled trial comparing radiologic pigtail-retained gastrostomy and radiologic mushroom-retained gastrostomy. J Vasc Interv Radiol 2017;28(12):1702-1707

9 Cantwell CP, Murray JG. Large-bore gastrostomy feeding tube insertion by a pull technique using a snare. Cardiovasc Intervent Radiol 2008;31(4):811-813

10 Narayanam S, Jaskolka J, Ho C. Percutaneous endoscopic gastrostomy (PEG) without endoscopy-a simplified technique of percutaneous trans-oral gastrostomy (PTOG). J Vasc Interv Radiol 2013;24(4):S154-S5

11 Cardella JF, Kundu S, Miller DL, Millward SF, Sacks D; Society of Interventional Radiology. Society of Interventional Radiology clinical practice guidelines. J Vasc Interv Radiol 2009;20(7, Suppl):S189-S191

12 Kuo YC, Shlansky-Goldberg RD, Mondschein JI, et al. Large or small bore, push or pull: a comparison of three classes of percutaneous fluoroscopic gastrostomy catheters. J Vasc Interv Radiol 2008;19(4):557-563, quiz 564

Erratum: This article was corrected as per erratum published on April 18, 2019 (DOI:10.1055/s-0039-1688684). 